\title{
Stimulated Multiphoton Bremsstrahlung in Electron-Ion Collisions*
}

\author{
Sydney Geltman** \\ Institute for Basic Standards, National Bureau of Standards, Boulder, Colorado 80302
}

(August 22, 1977)

\begin{abstract}
A review is presented of the basic classical mechanism whereby an electron can absorb energy from an electromagnetic field while undergoing a collision with an atom or ion. The process is also described for a quantum mechanical collision and the absorption or emission of photons. The appropriate formulations are described for weak and strong applied fields. A brief review is given for the resulting plasma absorption coefficients.
\end{abstract}

Key words: Bremsstrahlung; electron-ion collisions; free-free transitions; multiphoton processes; plasma absorption.

\section{Introduction}

A free electron colliding with an ion is accelerated in its potential field and on the basis of classical electrodynamics will emit electromagnetic radiation $[1,2] .{ }^{1}$ Such bremsstrahlung radiation has long been observed in collisions between high energy charged particles and nuclei. If an external radiation field is applied to an electron-ion system one has stimulated free-free transitions of the electron, corresponding to both the emission (bremsstrahlung) and absorption (inverse bremsstrahlung) of photons.

In a fully ionized plasma irradiated by a laser with frequency not near the plasma frequency, we can expect the above stimulated processes in electron-ion collisions to be the main mechanisms for the electron heating of the plasma. Electron-electron collisions do not lead to absorption of this type since that system does not have a dipole moment. Thus contributions of this type as well as of stimulated Compton scattering [3] will be of higher order in $\alpha$.

In this review we will confine ourselves to the nonrelativistic treatment of the electron motion, both classically and quantum mechanically. We treat the electromagnetic field entirely as a classical, monochromatic, coherent field, as we are not concerned with spontaneous emission or higher order quantum electrodynamic effects.

\section{Classical Picture}

In a harmonic, linearly polarized electric field, a free electron having drift or thermal velocity, $\mathbf{v}$, has the instantaneous resultant velocity

$$
\mathbf{w}(t)=\mathbf{v}+\mathbf{u}(t),
$$

* Based on a talk presented at the 2nd Conference on the Interaction of Electrons with Strong ectromagnetic Fields, Budapest, Hungary, October 6-10, 1975

** Staff Member, Quantum Physics Division at the Joint Institute for Laboratory Astrophysics, oulder, Colorado.

${ }^{1}$ Figures in brackets indicate the literature references at the end of this paper. where $\mathbf{u}(t)=\mathbf{u}_{0} \sin \omega t$ (with $\mathbf{u}_{0}=e \mathbf{E}_{0} / m \omega$ ) is the driven or quiver velocity which is imparted to the classical motion of an electron by the field [4]. If the electron did not make any collisions, its instantaneous kinetic energy would simply oscillate about its thermal value $1 / 2 m v^{2}$ and an assembly of such free electrons would have a temperature which remained constant in time.

A heating of such a plasma could occur only if there were a mechanism to convert some of the driven energy of the electrons into thermal energy, and this will occur if the oscillating electrons can make collisions with ions. In such a collision, characterized as occurring at time $t_{c}$, one can evaluate the thermal energy gain (or loss) by solving the classical equations of motion of the electron under the influence of both the applied radiation field and the ionic potential field, and determining

$$
\mathbf{w}\left(t_{c}+T\right)=\mathbf{v}_{f_{c}}+\mathbf{u}\left(t_{c}+T\right),
$$

subject to the initial condition

$$
\mathbf{w}\left(t_{c}-T\right)=\mathbf{v}_{i}+\mathbf{u}\left(t_{c}-T\right),
$$

with $T \rightarrow \infty$ being a valid idealization for large mean free time between collisions. The ion takes up a recoil momentum which corresponds to a very small part of the energy transfer (since $m / M \ll 1$ ) and the net thermal energy change of the electron, $\Delta \epsilon_{c}=1 / 2 m\left(v_{f c}^{2}-v_{i}^{2}\right)$, is considered to be taken from (or delivered to) the electromagnetic field.

The detailed solution of the classical equations of motion during a collision is quite complicated in general, so one makes the simplifying assumption that if the collision duration is much smaller than the applied period $\left(\tau_{c} \ll 1 / \omega\right)$ the scattering by the ion can be idealized as an instantaneous elastic deflection from $\mathbf{w}\left(t_{c}^{-}\right)=\mathbf{w}_{c}$ to $\mathbf{w}\left(t_{c}^{+}\right)=\mathbf{w}_{c}{ }_{c}$ (see fig. 1). In such an impact model the thermal energy change is

$$
\Delta \epsilon_{c}=\frac{1}{2} m\left(v_{f c}^{2}-v_{i}^{2}\right)=m \mathbf{u}_{c} \cdot\left(\mathbf{w}_{c}-\mathbf{w}_{c}^{\prime}\right),
$$




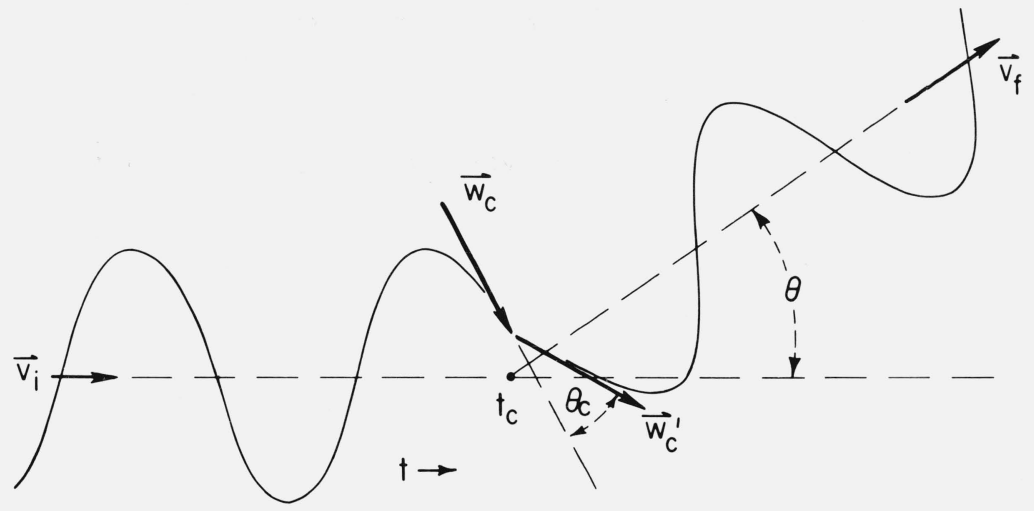

FIGURE 1. Trajectory of an electron in a strong oscillatory field in the region of an impact collison with an ion.

from which we see it depends upon the wave phase at the collision through $\mathbf{u}_{c}=\mathbf{u}_{0}$ sin $\omega t_{c}$, the angle between $\mathbf{w}_{c}$ and field direction, and the angle of scattering $\theta_{c}$. One can thus define a classical differential cross section for a free-free transition

$$
\frac{d \sigma^{\left(\Delta \epsilon_{c}\right)}}{d \Omega}=\frac{d \sigma_{e l}}{d \Omega_{c}}\left|\frac{d \Omega_{c}}{d \Omega}\right|
$$

where $d \Omega_{c} / d \Omega$ is a complicated kinematical factor relating the instantaneous impact vectors to laboratory fixed directions and the average is taken over all possible collisions compatible with the transition $\mathbf{v}_{i} \rightarrow \mathbf{v}_{f c}$. For applied fields which are so intense that $u_{0} \geq v_{i, f c}, d \Omega_{c} / d \Omega$ will become an extremely nonlinear function of $E_{0}$ and the impact energy $1 / 2$ $\boldsymbol{m} \boldsymbol{w}_{c}^{2}$ will vary strongly over a cycle of the field. If we go to the extreme weak-field case, where $u_{0} \ll v_{i}$, and low energy transfer, $\Delta \epsilon_{c} \ll \epsilon_{i}$, then impact energy $\cong \epsilon_{i}$ in (5), and it reduces to

$$
\frac{d \sigma^{\left(\Delta \epsilon_{c}\right)}}{d \Omega} \cong \frac{d \sigma_{e l}}{d \Omega}
$$

If we were now to assert that energy can be taken from the field in units of $\hbar \omega$ only, we could define a semi-classical $n$ photon free-free absorption cross section in terms of the various moments of $\left(\Delta \epsilon_{c}\right)^{2}$ by the correspondence:

$$
\left\langle\left(\Delta \epsilon_{c}\right)^{2}\right\rangle^{n} \frac{d \sigma_{e l}}{d \Omega} \cong(n \hbar \omega)^{2 n} \frac{d \sigma^{ \pm(n)}}{d \Omega} .
$$

If we carry this out for the single photon case, and average over all initial directions, we obtain the result of Zeldovich and Raizer [5]

$$
\frac{d \sigma^{ \pm(1)}}{d \Omega}=\frac{4}{3}\left(\frac{\epsilon_{i}}{\hbar \omega}\right)\left(\frac{\epsilon_{0}}{\hbar \omega}\right)(1-\cos \theta) \frac{d \sigma_{e l}}{d \Omega},
$$

where $\boldsymbol{\epsilon}_{0}=1 / 2 m u_{0}^{2}$. The above correspondence will give $n$ photon cross sections which are proportional to the $n$th power of the field intensity, which we know must be the case from the full quantum treatment, but in general so many approximations have gone into the above result that it has very limited use in quantitative applications. Furthermore, for the Coulomb case the angular integral of (8) will diverge unless there is a low-angle cut-off (or maximum impact parameter).

It is interesting to use this classical picture to estimate the rate of conversion of driven energy into thermal energy [6]. On the basis of the impact model the cycle-averaged time rate of energy transfer into transverse motion per electron is

$$
\begin{aligned}
\frac{d}{d t}\left\langle\Delta \epsilon_{c \perp}\right\rangle & =m N_{i}\left\langle u_{c} \int d \Omega \frac{d \sigma_{e \ell}}{d \Omega} \mathbf{u}_{c} \cdot\left(\mathbf{w}_{c}-\mathbf{w}_{c}{ }^{\prime \prime}\right)\right\rangle \\
& =m N_{i}\left\langle u_{c} \mathbf{u}_{c} \cdot \mathbf{w}_{c} \sigma_{d}\left(\mathbf{w}_{c}\right)\right\rangle .
\end{aligned}
$$

The momentum transfer cross section $\sigma_{\boldsymbol{d}}$ for a Coulomb potential has the velocity dependence $\sigma_{d} \sim w_{c}^{-4}$, and if one averages over all directions of $\mathbf{w}_{c}$ one has

$$
\frac{d}{d t}\left\langle\Delta \epsilon_{c \perp}\right\rangle=A\left\langle\frac{u_{c}^{2}}{\left(u_{c}^{2}+w_{\perp}^{2}\right)^{3 / 2}}\right\rangle
$$

where $\mathbf{w}_{c}$ has been resolved into components along $\left(u_{c}\right)$ and transverse to the field $\left(w_{\perp}\right)$. Thus the average over phase of the field cycle is

$$
\frac{d}{d t}\left\langle\Delta \epsilon_{c \perp}\right\rangle=\frac{A}{u_{0}}\left(\frac{2 \omega}{\pi}\right) \int_{0}^{\pi / 2 \omega} d t_{c} \frac{\sin ^{2} \omega t_{c}}{\left(\sin ^{2} \omega t_{c}+w_{\perp}^{2} / u_{0}^{2}\right)^{3 / 2}},
$$

and the rate of conversion of the ordered, driven motion into the transverse, thermal motion goes as $u_{0} / w_{\perp}{ }^{3}$ for $u_{0}<<$ 
$w_{\perp}$, and as $1 / u_{0}$ for $u_{0} \gg w_{\perp}$. In figure 2 we have curves indicating this behavior. Thus in the limit of strong fields there is a reduction with increasing field in the effective free-free absorption. This is due to the decrease in $\sigma_{d}$ with increasing impact velocity. We will see similar behavior in the quantum description.

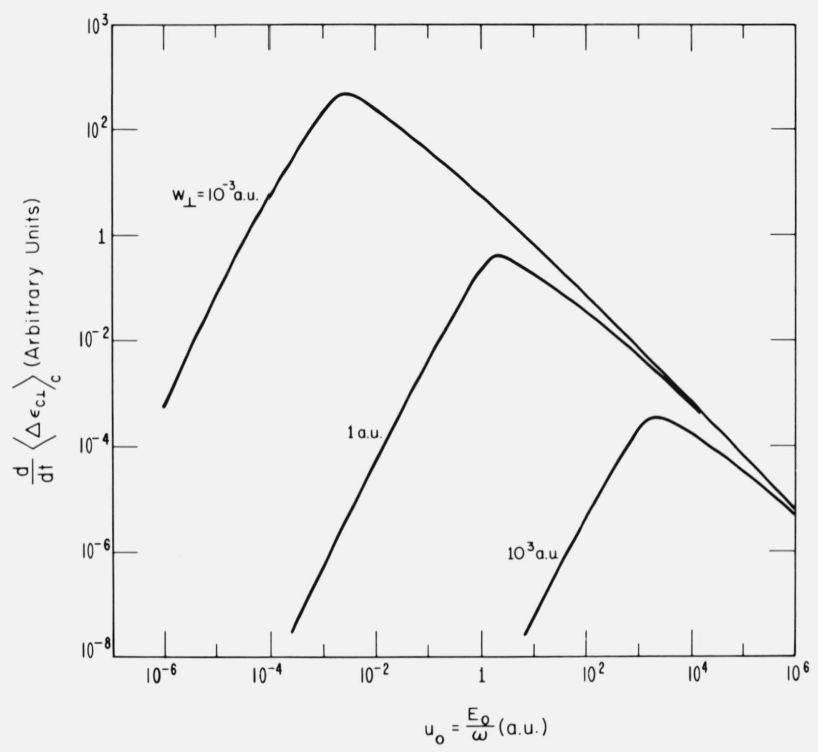

FIGURE 2. Cycle-averaged rate of classical energy transfer from driven motion into thermal motion.

A more complete classical treatment of the nonlinear effects of strong fields on plasma properties was carried out by Silin [7]. The nonlinear dependence of the current density on the electric field strength introduces higher harmonics in the effective field, which can be interpreted as multiphoton phenomena.

\section{Quantum Description}

From the preceding it is seen that the available classical description of free-free absorption is useful for an intuitive physical picture of the process, but is extremely limited as quantitative theory. For example, while the impact model is adequate for microwave absorption it would break down for slow electrons $(<1 \mathrm{eV})$ in visible wavelengths. Also semiclassical results such as (7) or (8) will lead to divergent total free-free cross sections in the Coulomb field unless cut-offs are applied. For a complete description it is necessary to proceed to the quantum formulation.

The quantum problem is formulated by first considering the electron-ion scattering problem in the absence of a radiation field, where the initial stationary state of the electron is

$$
\psi_{\mathbf{k}_{i}}(\mathbf{r}, t)=u_{\mathbf{k}_{i}}(\mathbf{r}) e^{-i_{\epsilon_{i}} t / \hbar}
$$

We assume that the laser field is switched on from $t=0$ to $T$, and wish to find the cross section for finding the electron in the final stationary state for $t>T$,

$$
\psi_{\mathbf{k}_{f}}(\mathbf{r}, t)=u_{\mathbf{k}_{f}}(\mathbf{r}) e^{-i \boldsymbol{i}_{f} t / \hbar}
$$

A solution of the full Schrödinger equation in $0 \leq t \leq T$ subject to initial condition (12), leads to the transition rate

$$
W_{i f}=\lim _{T \rightarrow \infty} \frac{1}{\mathrm{~T}}\left|\left\langle\psi_{\mathbf{k}_{f}} \mid \Psi_{i}(\mathbf{r}, T)\right\rangle\right|^{2}
$$

The use of quantum theory to describe the electronic motion results in the appearance of energy conserving $\delta\left(\epsilon_{f}-\epsilon_{i} \mp\right.$ $n \hbar \omega)$ functions in $W_{i f}$, and the required summation over final states $\left(\epsilon_{f}\right)$, gives the transition rates for absorption or emission of $n$ quanta,

$$
\begin{aligned}
\sum_{\epsilon_{f}} \boldsymbol{W}_{i f}=\frac{\mathcal{V}}{(2 \pi)^{3}} \int_{0}^{\infty} d k_{f} k_{f}^{2} W_{i f} & \\
& \equiv \sum_{n=1}^{\infty}\left[S_{n}\left(\mathbf{k}_{i} \rightarrow \hat{\mathbf{k}}_{f}\right)+S_{-n}\left(\mathbf{k}_{i} \rightarrow \hat{\mathbf{k}}_{f}\right)\right] .
\end{aligned}
$$

where $V$ is a quantization volume. The $n$-photon transition rates are then related to a differential cross section for $n$ photon absorption (emission) by dividing by the incident electron flux,

$$
\frac{d \sigma^{ \pm(n)}}{d \Omega}=\left(\frac{m V}{\hbar k_{i}}\right) S_{ \pm n}\left(\mathbf{k}_{i} \rightarrow \hat{\mathbf{k}}_{f}\right)
$$

In practice it is not possible to exactly solve the full Schrödinger equation while the field is applied, so we must use a perturbation procedure. There are two ways of splitting the full Hamiltonian,

$$
H=\frac{p^{2}}{2 m}+V(\mathbf{r})-\frac{e}{m c} \mathbf{p} \cdot \mathbf{A}(t)
$$

(in the dipole approximation the $A^{2}$ term is removed by a unitary transformation) depending on whether the applied field is weak or strong, i.e., the relative average magnitudes of the $V$ and $\mathbf{p} \cdot \mathbf{A}$ terms.

\subsection{Weak Fields}

Before the advent of high power lasers all laboratory radiation sources provided very weak fields compared with atomic potential fields. For example, one megawatt $/ \mathrm{cm}^{2}$ of laser radiation attains an $E_{0}$ of only about $10^{-5}$ of an atomic unit. Thus all of the conventional atomic radiation theory [7] is formulated by using $H_{0}=\frac{p^{2}}{2 m}+V$ as the unperturbed part of (17) and $H^{\prime}=-\frac{e}{m c} \mathbf{p} \cdot \mathbf{A}$ as the perturbation. As is well known, successive iterations of the time-dependent Schrödinger equation lead to improved approximations for $\Psi_{i}(\mathbf{r}, T)$, and consequently for $d \sigma^{ \pm(n)} / d \Omega$. In the limit of an infinite number of iterations the cross section takes the form (in atomic units) 


$$
\frac{d \sigma^{ \pm(n)}}{d \Omega}=\frac{a_{0}^{2}}{8 \pi^{2}} \frac{k_{f}}{k_{i}}\left|\sum_{m=0}^{\infty}\left(\frac{E_{0}}{2 \omega}\right)^{n+2 m} \sum_{j} D_{n m j}\right|^{2}
$$

where $D_{n m j}$ is the $j$ th "diagram" and $m$ is the number of "virtual photon pairs." We will not go into the details of the construction and enumeration of these diagrams, but rather simply give the following typical example [8,9]:

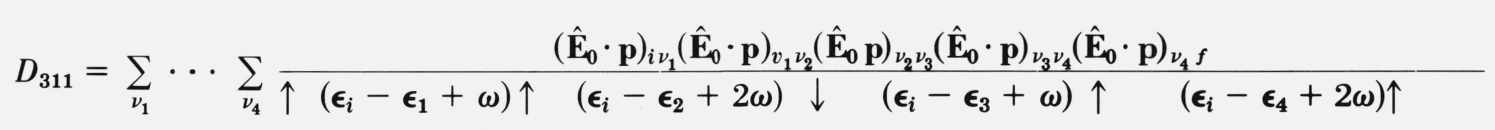

where the sums include all bound and continuum states of the electron-ion system and the arrows below indicate absorptions or emissions of photons, thereby providing a pictorial representation for the "diagram."

To date there have been extensive calculations $[10,11$, $12,13]$ on the lowest order single-photon free-free absorption of an electron in a Coulomb field. The angle-integrated and polarization-averaged result can be written as

$$
\sigma^{ \pm(1)}=\sigma_{K} g_{F F}\left(k_{i}, k_{f}\right),
$$

where

$$
\sigma_{K}=\frac{\pi^{2}}{\sqrt{3}} \alpha^{2} \frac{c e^{2} Z^{2} E_{0}^{2}}{m \hbar \omega^{4} k_{i}}
$$

is a cross section originally derived semi-classically by Kramers [14] and $g_{F F}$ is the Coulomb quantum correction known as the Gaunt factor.

There appears to have been no theoretical work done on weak-field multiphoton free-free processes. The lack of motivation is understandable since such processes are of order $\left(E_{0} / 2 \omega\right)^{2 n}$ and thus a negligible contribution (compared with $n=1$ ) to any continuous absorption by the plasma. On the other hand many multiphoton bound-bound and boundfree calculations have been done, as in those cases the multiphoton process could be the dominant one.

From expression (18) we see that the free-free cross section arising from weak-field perturbation theory is essentially a power series in $E_{0} / 2 \omega$. Since we have no a priori knowledge of the variation of $\sum_{j} D_{n m j}$ with $m$, we can't make any statements about the absolute convergence of this expansion, but a reasonable guess is that it is an asymptotic series for any value of $E_{0}$. It clearly is not a useful expansion for the very strong field situation where $E_{0} / 2 \omega \geqslant 1$ a.u.

\subsection{Strong Fields}

When the magnitudes of the applied fields are comparable with or greater than the mean atomic field it becomes necessary to alter the choices of the unperturbed and perturbation parts of $H$ in the approximate evaluation of $\Psi_{i}(\mathbf{r}, T)[14,15,16]$. One now chooses $H_{0}=\frac{p^{2}}{2 m}-\frac{e}{m c}$ $\mathbf{p} \cdot \mathbf{A}$, and the ionic potential $V$ is regarded as the perturbation in the interval $0 \leq t \leq T$. The unperturbed wave functions are

$$
\begin{aligned}
\phi_{\mathbf{k}}(\mathbf{r}, t)=\exp \{i[\mathbf{k} \cdot \mathbf{r} \\
\left.\left.-\frac{\hbar}{2 m} \int_{0}^{t} d \tau\left(k^{2}-\frac{2 e}{\hbar c} \mathbf{k} \cdot \mathbf{A}(\tau)\right)\right]\right\}
\end{aligned}
$$

which are not stationary states, but rather represent the quivering motion of a classical electron in the oscillating field. As a result of a single iteration of the time-dependent Schrödinger equation, using the Green's function appropriate to basis functions (22) and going through the steps indicated in (14)-(16), one obtains the free-free cross sections

$$
\begin{aligned}
\frac{d \sigma^{ \pm(n)}}{d \Omega}= & (2 \pi)^{10} a_{0}^{2} \frac{k_{f}}{k_{i}} \iint d \mathbf{p} d \mathbf{q} \cdot \\
& \cdot\left|\tilde{u}_{i}(\mathbf{p}) \frac{\tilde{V}(\mathbf{p}-\mathbf{q})}{e^{2} a_{0}^{2}} \tilde{u}_{f}^{*}(\mathbf{q}) J_{n}\left(x_{p q}\right)\right|^{2}
\end{aligned}
$$

where the Fourier transform is $\tilde{u}(\mathbf{p})=(2 \pi)^{-3} \int d \mathbf{r} e^{-i \mathbf{p} \cdot \mathbf{r}} u(\mathbf{r})$, $x_{\boldsymbol{p q}}=\boldsymbol{\beta} \cdot(\mathbf{q}-\mathbf{p})$, and $\boldsymbol{\beta}=\frac{e \mathbf{E}_{0}}{m \omega^{2}}$ is the maximum classical displacement of a free electron in the applied field. The Bessel functions arise from an expansion of $e^{i \boldsymbol{\beta} \cdot(\boldsymbol{q}-\boldsymbol{p}) \sin \omega t}$, which enters through the functions (22). Carrying out higher iterations would give an expression containing higher powers of $\hat{V}$, similar to a Born expansion for the elastic scattering cross section. (Recall that the first Born approximation to $d \sigma_{e l} / d \Omega$ is proportional to $|\hat{V}|^{2}$.) In fact Kroll and Watson $[17,18,19]$ have carried out this iteration, and have obtained the following approximate result

$$
\frac{d \sigma^{ \pm(n)}}{d \Omega} \cong \frac{k_{f}}{k_{i}} J_{n}^{2}\left(x_{i f}\right) \frac{d \sigma_{e l}(\overline{\boldsymbol{\epsilon}}, \mathbf{Q})}{d \Omega},
$$

where $\overline{\boldsymbol{\epsilon}}$ is the mean incident energy $\hbar^{2} / 2 m\left(\mathbf{k}_{i}+\hat{\mathbf{E}}_{\mathbf{0}} m n \omega\right)$ $\left.\hat{\mathbf{E}}_{\mathbf{0}} \cdot \mathbf{Q}\right)^{\mathbf{2}}$ and $\mathbf{Q}=\mathbf{k}_{f}-\mathbf{k}_{i}$ is the actual momentum change. Since $\left|\mathbf{k}_{f}\right| \neq\left|\mathbf{k}_{i}\right|$ when photons are absorbed or emitted, the actual scattering angle corresponding to the momentum change $\mathbf{Q}$ is given by $\cos \theta=1-\hbar^{2} Q^{2} / 2 m \bar{\epsilon}$. Since $d \sigma_{e l} / d \Omega$ appears explicitly in (24), as it did in the classical expressions (5), the Kroll-Watson result is basically an impact approximation and thus applicable unly at low frequencies.

If plane-wave initial and final scattering functions are used in (23) it reduces to 


$$
\frac{d \sigma^{ \pm(n)}}{d \Omega}=4 a_{0}^{2} \frac{k_{f}}{k_{i}} \frac{Z^{2}}{\left(Q a_{0}\right)^{4}} J_{n}^{2}\left(x_{i f}\right)
$$

for the case of the Coulomb potential. A more precise representation [20] of the scattering wave functions in a Coulomb field leads to the additional factor for (25),

$$
f_{c}=81\left[\left(\eta_{i}^{2}+1\right)\left(\eta_{i}^{2}+9\right)\left(\eta_{f}^{2}+1\right)\left(\eta_{f}^{2}+9\right)\right]^{-1}
$$

where $\eta_{i}=-Z / k_{i} a_{0}$ and $\eta_{f}=-Z / k_{f} a_{0}$. A thorough analysis of the angular distributions contained in (25) was made by Elyutin [21] for various magnitudes of the quantities $E_{0} v_{i} / \omega^{2}$ and $E_{0} / v_{i} \omega$ (in atomic units), and it is found that under some conditions and angles the emission cross section exceeds the absorption cross section. Brehme [22] has done extensive numerical calculations on the angular integral of (25), and some of these results are shown in figure 3 as a function of photon flux. Also shown in this figure is the weak-field single-photon absorption cross section which is obtained from (20). The photon flux at which $E_{0} / \omega=1$ a.u. is indicated, and the exact result for $\sigma^{+(1)}$ is expected to join smoothly between these weak and strong-field theories. The wiggles in the strong-field curves arise from the $J_{n}^{2}$ factor, and one sees a primary maximum followed by a downward trend with increasing radiation intensity. This behavior follows from the asymptotic envalope of $J_{n}^{2}(x) \sim$ $E_{0}^{-1} \sim \rho^{-1 / 2}$. This is qualitatively similar to the classical fall-off of the rate of thermal energy increase which is shown in figure 2 .

We note that the strong-field formulation of free-free absorption gives expressions which are analytic functions of the field strength $E_{0}$, unlike the power series form of the weak-field perturbation theory. Thus it seems likely that higher iterations of the strong-field method will give satisfactory values for the cross sections in the intermediate region, where $E_{0} / \omega \sim 1$ a.u. Pert [23] has shown that (25) for $n=1$ goes over to the semiclassical (8) in the low-field limit after averaging over oscillations of the Bessel function. He has also shown the correspondence between (25) and the purely classical treatment (5) using a method due to Rand [24].

The first direct observation of a laser-induced free-free multiphoton process has been very recently reported by Weingartshofer et al. [25]. They observed the absorption and emission of $\mathrm{CO}_{2}$ laser photons by an electron beam undergoing large-angle scattering by argon atoms. A near monoenergetic beam of $11 \mathrm{eV}$ electrons was scattered by argon atoms in the presence of a pulsed $\mathrm{CO}_{2}$ laser producing a radiation flux density of $10^{9} \mathrm{~W} / \mathrm{cm}^{2}$. The electrons scattered through an angle of $153^{\circ}$ were detected and energy analyzed with laser off and on. The results of counting over many laser pulses are shown in figure 4 in terms of scattered electron current (in arbitrary units) for fixed incident current plotted against scattered electron energy in units of laser photons $(0.117 \mathrm{eV})$. One clearly sees the distinct peaks representing the absorption and emission of up to three photons, with their peak values approximately satisfying (24). The widths are a result of the energy width of the incident electron beam and the energy resolution of the spectrometer $(0.055 \mathrm{eV})$. Since the energy gained or lost by the electrons is very small compared with their initial

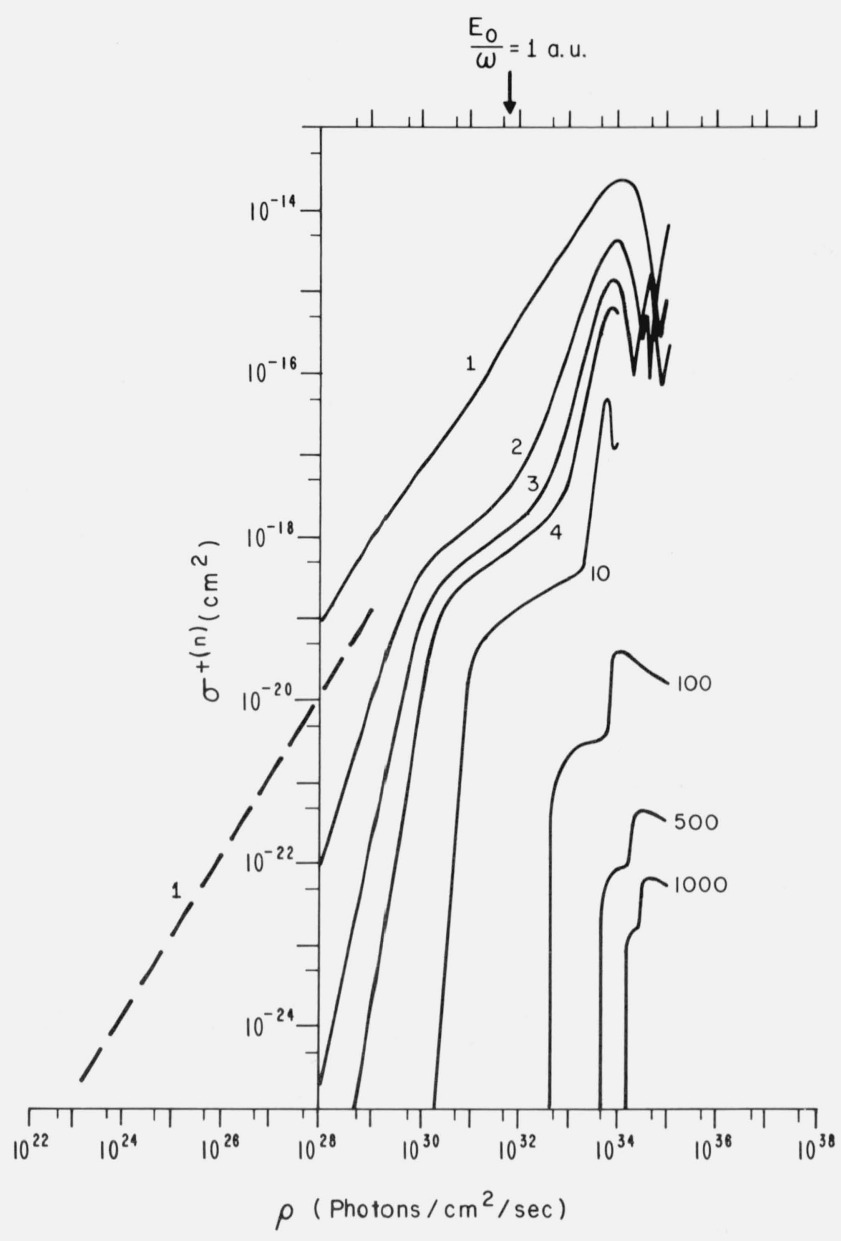

Figure 3. Total cross section for the strong-field n-photon absorption (ruby laser $\hbar \omega=1.8 \mathrm{eV}$ ) by an electron-proton system in a $100 \mathrm{eV}$ scattering state (solid lines); corresponding weak-field 1-photon absorption (dashed line).

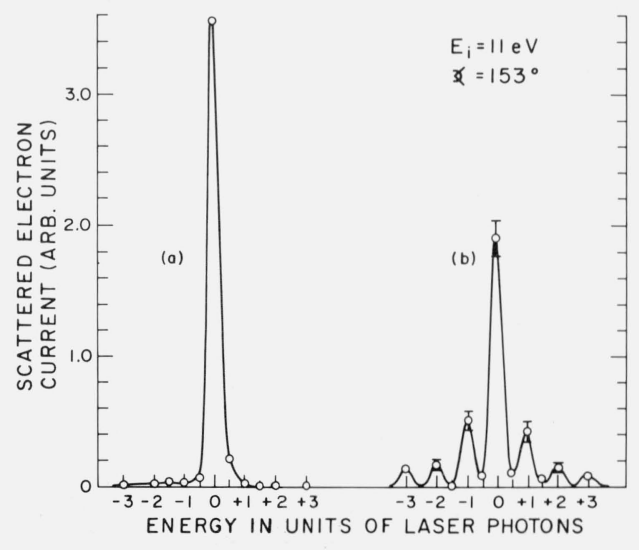

FiguRE 4. Weingartshofer et al. [25] data on free-free transitions of electrons scattered from argon atoms. Integrated current with (a) laser off and (b) laser on. 
energy, $x_{i f}$ is essentially a constant, and using the identity

$$
\sum_{n=-\infty}^{\infty} J_{n}^{2}(x)=1
$$

one has from (24) that

$$
\sum_{n} \frac{d \sigma^{ \pm(n)}}{d \Omega}=\frac{d \sigma_{e l}}{d \Omega}
$$

[like classical result (6)]. It is obvious from figure 4 that this sum rule is well satisfied.

\section{Plasma Averages}

In the last two sections we have reviewed the stimulated absorption and emission of multiphoton radiation by an electron in an encounter with a single ion. When one goes over to the case of a real plasma it is clear that the macroscopic parameters of density and temperature must also be considered in the overall description of the absorption of external laser radiation. If these are such that an electron makes many collisions in the course of a cycle of the field $\left(2 \pi N_{i} \beta^{2} v / \omega>1\right)$, the properties of the plasma cannot be completely analyzed in terms of binary collision cross sections alone.

Hughes and Nicholson-Florence [26] have studied the heating of a plasma in the weak field case by single photon absorption and emission. They have used form (20) for the cross sections and have investigated absorption coefficients for both thermal and non-thermal velocity distributions. They have obtained numerical results for power absorbed by the plasma, but appear to go to such high incident power fluxes that would require use of cross section form (25) rather than (20). Nicholson-Florence [27] used (25) including both stimulated absorption and emission for the $n=1,2,3$, 4 cases to numerically evaluate the expected power absorption for a wide range of the parameters $\xi=\hbar \omega / m v^{2}$ and $\gamma$ $=e v E_{0} / \hbar \omega^{2}$. The results typically show a maximum in the power absorption versus $\gamma$ similar to those seen in figures 2 and 3.

Seeley and Harris [28] have used (25) and the zerothorder assumption of a Maxwellian velocity distribution to evaluate the time derivative of a first order velocity distribution function for weak and strong fields, and obtain results in essential agreement with Silin's classical results [7]. They find that for weak fields only single-photon processes are important while at strong fields multiphoton processes are dominant, with the most important $n$ values given by $n \hbar \omega \cong$ $\frac{1}{2} m u_{0}^{2}$, the peak classical driven energy. For the weak fields they should have used cross section form (20) for $\sigma^{1}$, but from figure 3 we see that the error made is less than an order of magnitude. This would probably not affect their conclusions, particularly since it is expected that weak field $d \sigma^{ \pm(n)} / \mathrm{d} \Omega(n>1)$ are even further below the respective values that would result from (25).

A study of the departure of the total strong-field absorption coefficient of a plasma from its single-photon value was made by Osborn [29]. The total rate per unit volume for the absorption or emission of $n$ photons is given by

$$
R_{ \pm n}=N_{e} N_{i} \int d \mathbf{v} f(\mathbf{v}) v \sigma^{ \pm(n)}
$$

The distribution function $f(\mathbf{v})$ is assumed to be Maxwellian and the strong-field cross section (25) is taken for $\sigma^{ \pm(n)}$. The net energy absorption rate is now

$$
\alpha=\sum_{n=1}^{\infty} n \hbar \omega\left(R_{+n}-R_{-n}\right) \equiv \alpha_{1} F(x, y)
$$

where $\alpha_{1}$ is the single-photon value

$$
\alpha_{1}=\hbar \omega\left(R_{+1}-R_{-1}\right) .
$$

The parameters in the coefficient $F$ are $x=1 / 2 m u_{0}^{2} / 2 k T$ and $y=\hbar \omega / 2 k T$, i.e., essentially ratios of driven energy and photon energy to mean thermal energy. In the limit of $x$, $y \rightarrow 0, F \rightarrow 1$, or the entire absorption is due to singlephoton processes. Brysk [30] has carried out a more detailed analysis of $F(x, 0)$ and the result is given in figure 5 . The large reduction in $\alpha$ from $\alpha_{1}$ shown here for large values of $x$ implies that stimulated emission in multiphoton transitions is more important (relative to stimulated absorption) than in the single photon case.

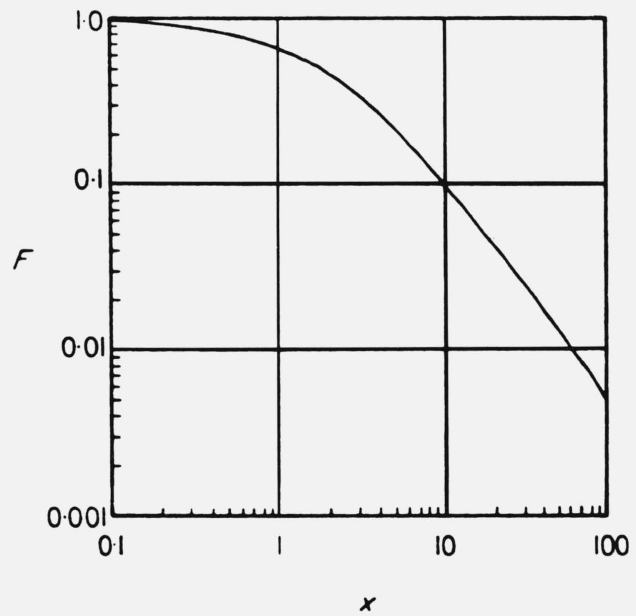

FIGURE 5. Factor representing multiphoton contribution to total free-free absorption coefficient.

\section{Conclusions}

The weak- and strong-field forms of the nonrelativistic free-free problem apear to be well understood and are being applied to the calculation of absorption coefficients for actual plasma conditions. The general behavior of the cross sections as a function of radiation flux is an increasing function at low fluxes and an oscillating function with decreasing envelope at large fluxes, with a maximum in the region where the magnitude of the applied electric field 
equals the mean atomic field strength. Similarly the absorption coefficient of the plasma due to inverse bremsstrahlung will have a maximum as a function of applied field strength at any given temperature, indicating that there exists an optimum laser intensity for maximum efficiency in heating the plasma.

Relativistic effects have so far been largely neglected in the theory on the grounds that they would probably not alter the qualitative absorption characteristics [6, 22]. However, since the maximum driven velocity $u_{0}=e E_{0} / m \omega$ becomes equal to $c$ at a Nd-glass laser intensity of $6 \times 10^{17} \mathrm{~W} / \mathrm{cm}^{2}$, it would be worthwhile to make a more careful estimate of relativistic effects.

Our remarks above have been confined to the case of linearly polarized radiation. However, a study [31] shows that the absorption rate does not depend on the polarization in the weak-field case but does become very dependent on the polarization in the strong-field case.

In concluding, we would like to bring to the reader's attention a very recent bibliography [32] and a review [33] on free-free transition processes involving electron collisons on neutral atoms.

\section{References}

[1] Landau, L. D., and Lifshitz, E. M., The Classical Theory of Fields (Addison-Wesley 1962, Chapter 9).

[2] Jackson, J. D., Classical Electrodynamics (John Wiley 1962, Chapter $15)$.

[3] Bunkin, F. V., Kazakov, A. E., and Federov, M. V., Usp. Fiz. Nauk, 107, 559 (1972); [Sov. Phys.-Usp. 15, 416 (1973)].

[4] Pert, G. J., J. Phys. A: Gen. Phys. 5, 506 (1972).

[5] Zeldovich, Ya. B., and Raizer, Yu. P., Zh. Eksp. Theor. Fiz. 47, 1150 (1964); [Sov. Phys.-JETP 20, 772 (1965)].
[6] Bethe, H. A., Los Alamos Scientific Laboratory Informal Report No. LA-5031-MS, 1972 (unpublished).

[7] Silin, V. P., Zh. Eksp. Teor. Fiz. 47, 2254 (1964); [Sov. Phys.JETP 20, 1510 (1965)].

[8] Heitler, W., The Quantum Theory of Radiation (Third Ed., Oxford, 1954).

[9] Gontier, Y., Rahman, N. K., and Trahin, M., Phys. Rev. Lett. 34, 779 (1975).

[10] Gaunt, J., Philos. Trans. Roy. Soc. London A229, 163 (1930).

[11] Sommerfeld, A., Atombau und Spektrallinien II (Vieweg, 1951).

[12] Karzas, W. J., and Latter, R., Astrophys. J. Suppl. 6, 167 (1961).

[13] Peach, G., Mem. R. Astro. Soc. 71, 1 (1967).

[14] Johnston, R. R., J.Q.S.R.T. 7, 815 (1967).

[15] Bunkin, F. V., and Federov, M. V., Zh. Eksp. Teor. Fiz. 49, 1215 (1965); [Sov. Phys.-JETP 22, 884 (1966)].

[16] Geltman, S., and Teague, M. R., J. Phys. B: Atom. Molec. Phys. 7, L22 (1974).

[17] Kroll, N. M., and Watson, K. M., Phys. Rev. A 8, 804 (1973).

[18] Rahman, N. K., Phys. Rev. A 10, 440 (1974).

[19] Choudhury, B. J., Phys. Rev. A 11, 2194 (1975).

[20] Geltman, S., J. Phys. B: Atom. Molec. Phys. 8, L374 (1975).

[21] Elyutin, P. V., Zh. Eksp. Teor. Fiz. 65 (1973); [Sov. Phys.-JETP 38, 1097 (1974)].

[22] Brehme, H., Phys. Rev. C 3, 837 (1971).

[23] Pert, G. J., J. Phys. A: Gen. Phys. 5, 1221 (1972).

[24] Rand, S., Phys. Rev. 136, B231 (1964).

[25] Weingartshofer, A., Holmes, J. K., Caudle, G., Clark, E. M., and Krüger, H., Phys. Rev. Lett. 39, 269 (1977).

[26] Hughes, T. P., and Nicholson-Florence, M. B., J. Phys. A. (Proc. Phys. Soc.) 1, 588 (1968)

[27] Nicholson-Florence, M. B., J. Phys. A: Gen. Phys. 4, 574 (1971).

[28] Seely, J. F., and Harris, E. G., Phys. Rev. A 7, 1064 (1973).

[29] Osborn, R. K., Phys. Rev. A 5, 1660 (1972).

[30] Brysk, H., J. Phys. A: Math. Gen. 8, 1260 (1975).

[31] Shima, Y., and Yatom, H., Phys. Rev. A 12, 2106 (1975).

[32] Gallagher, J. W., Bibliography of Free-Free Transitions in Atoms and Molecules, JILA Information Center Report No. 16 (JILA, University of Colorado, Boulder, 1977).

[33] Gavrila, M., in International Conference on the Physics of Electronic and Atomic Collisons. 10th. Invited Papers (North-Holland, Amsterdam, in press). 